1 Hacettepe Journal of Mathematics and Statistics

$\bigcap$ Volume 45 (5) (2016), 1343-1354

\title{
Fibonacci, and Lucas Pascal triangles
}

\author{
Hacène Belbachir* and László Szalay ${ }^{\dagger \ddagger}$
}

\begin{abstract}
In this paper, we give explicit formulas for elements of the Fibonacci, and Lucas Pascal triangles. The structure of these objects and Pascal's original triangle coincide. Keeping the rule of addition, we replace both legs of the Pascal triangle by the Fibonacci sequence, and the Lucas sequence, respectively. At the end of the study we describe how to determine such a formula for any binary recurrence $\left\{G_{n}\right\}_{n=0}^{\infty}$ satisfying $G_{n}=G_{n-1}+G_{n-2}$. Other scattered results are also presented.
\end{abstract}

Keywords: Generalized Pascal's triangle, Fibonacci sequence, Lucas sequence.

2000 AMS Classification: 11B39, 05B30

Received: 07.07.2015 Accepted : 08.12.2015 Doi : 10.15672/HJMS.20164515688

\section{Introduction}

Although a lot is known about the Pascal triangle, its origin is lost in the mist of time. Since the work of Pascal [10] several scholars have contributed with variations, generalizations to this object. An early generalization is due to Raab [11], who introduced the so-called $A B$-based Pascal triangles. Its structure is identical to the regular Pascal triangle, and the elements are the coefficients of $x^{n-k} y^{k}$ in the expansion of the polynomial $(A x+B y)^{n}$. Some variations, for instance the Pascal pyramid, stem from different combinatorial approaches. The Hosoya's triangle [7] is also a triangular arrangement based on the Fibonacci numbers, where each entry is the sum of the two entries above in either the left diagonal or the right diagonal. Koshy [9] gave a description on different Pascal-like triangles which are linked to the sums $\alpha^{n}+\beta^{n}$ (and the differences $\alpha^{n}-\beta^{n}$ ), where $\alpha$ and $\beta$ are the zeros of the characteristic polynomial $x^{2}-A x-B$ of the linear recurrence $G_{n}=A G_{n-1}+B G_{n-2}$. Sun [12] provided a generalization of the $D F F$, and $D F F z$ triangles introduced by Ferri et al. [5,6], respectively. Generally, the cited papers

*USTHB, Faculty of Mathematics, Recits Laboratory, Algiers, Algeria.

Email: hacenebelbachir@gmail.com

${ }^{\dagger}$ J. Selye University, Department of Mathematics and Informatics, Komarno, Slovakia.

Email: szalay.laszlo@nyme.hu

$\ddagger$ Corresponding Author. 
work with elementary considerations, using the properties of binomial coefficients and certain sequences.

In this paper, we keep the arrangement of Pascal's original triangle and, apart from the beginning, the rule of addition, but we vary the sequences located on the legs of the triangle. More precise description will be given later. The main purpose of this work is to give applicable explicit formulas for the elements of the so-called Fibonacci, and Lucas Pascal triangle. Note that the Fibonacci triangle studied by the present paper and Hosoya's triangle do not coincide since the insertion methods are different. Ensley [4] already derived a formula for the elements of Fibonacci Pascal triangle. While his result is given by a weighted sum of certain binomial coefficents, here (in Corollary 6) the exponential and polynomial terms are separated. The principal results are Theorems 1 and 4 in Section 3. As a consequence of these outcomes, we are able to determine analogous formula for the triangle generated by any binary recurrence $\left\{G_{n}\right\}_{n=0}^{\infty}$ satisfying $G_{n}=G_{n-1}+G_{n-2}$ (Theorem 5). On the other hand, we provide certain interesting arguments on arithmetic triangles, some of them have been justified previously. In fact, such objects are actually very popular, and one can find lot of information about them in the literature (see $[2,3,4,12]$ ).

Let $\left\{a_{n}\right\}_{n=0}^{\infty}$ and $\left\{b_{n}\right\}_{n=0}^{\infty}$ denote two real sequences. There is no importance if $a_{0} \neq b_{0}$, in this case we replace both terms by $\Omega$ as an indeterminate object.

The two initial sequences, as it was described by Dil and Mező [3], generate an infinite matrix $\mathbf{M}=\left(M_{k, n}\right)_{k \geq 0, n \geq 0}$ as follows. Put $M_{0,0}=\Omega$, and

$$
M_{k, 0}=a_{k}, \quad M_{0, n}=b_{n}, \quad k \geq 1, n \geq 1,
$$

further let

$$
M_{k, n}=M_{k, n-1}+M_{k-1, n}, \quad k n \neq 0 .
$$

For $k \geq 1$ and $n \geq 1$ the authors proved the explicit formula

$$
M_{k, n}=\sum_{i=1}^{k}\left(\begin{array}{c}
k+n-i-1 \\
n-1
\end{array}\right) a_{i}+\sum_{j=1}^{n}\left(\begin{array}{c}
k+n-j-1 \\
k-1
\end{array}\right) b_{j} .
$$

A similar approach in constructing a sort of Generalized Arithmetic Triangle (in short GAT) was used in [2]. Letting $A, B \in \mathbb{R}$, the GAT is structurally identical with Pascal's original triangle (Pascal himself called his object arithmetic triangle) and contains rows numbered by $0,1,2, \ldots$ such that the $n^{\text {th }}$ row possesses the elements $\left\langle\begin{array}{l}n \\ k\end{array}\right\rangle$ in the positions (say columns) $k=0,1, \ldots, n$ as follows.

Let $\left\langle\begin{array}{l}0 \\ 0\end{array}\right\rangle$ be arbitrary denoted by $\Omega$, and for positive integer $n$ put $\left\langle\begin{array}{l}n \\ 0\end{array}\right\rangle=A^{n} a_{n}$ and $\left\langle\begin{array}{l}n \\ n\end{array}\right\rangle=B^{n} b_{n}$, further for $n \geq 2$ and $1 \leq k \leq n-1$ let

$$
\left\langle\begin{array}{l}
n \\
k
\end{array}\right\rangle=B\left\langle\begin{array}{l}
n-1 \\
k-1
\end{array}\right\rangle+A\left\langle\begin{array}{c}
n-1 \\
k
\end{array}\right\rangle \text {. }
$$

Theorem 1 of [2] admits the direct formula

$$
\left\langle\begin{array}{l}
n \\
k
\end{array}\right\rangle=A^{n-k} B^{k}\left(\sum_{i=1}^{n-k}\left(\begin{array}{c}
n-1-i \\
k-1
\end{array}\right)_{\Gamma} a_{i}+\sum_{j=1}^{k}\left(\begin{array}{c}
n-1-j \\
n-k-1
\end{array}\right)_{\Gamma} b_{j}\right),
$$

to express $\left\langle\begin{array}{l}n \\ k\end{array}\right\rangle$ in the terms of $A, B$ and the sequences if $1 \leq n$ and $0 \leq k \leq n$. The extension $(\cdot)_{\Gamma}$ of binomial coefficients to arbitrary integers $n$ and $k$ appeared in (1.2) is obtained by the Gamma function (see [1], formula 6.1.21):

$$
\left(\begin{array}{l}
n \\
k
\end{array}\right)_{\Gamma}=\lim _{n_{1} \rightarrow n} \lim _{k_{1} \rightarrow k} \frac{\Gamma\left(n_{1}+1\right)}{\Gamma\left(k_{1}+1\right) \cdot \Gamma\left(n_{1}-k_{1}+1\right)} .
$$


We really need it since if $k=0$ or $k=n$ in (1.2), then the lower index of the binomial coefficients is negative. Note that $\left(\begin{array}{l}n \\ k\end{array}\right)_{\Gamma}=\left(\begin{array}{l}n \\ k\end{array}\right)$ if $k$ is nonnegative.

Our Generalized Aritmetic Triangle extends Ensley's GAT [4], since here we allow $a_{0} \neq b_{0}$ in the generator sequences, further we also vary the rule of addition by the parameters $A$ and $B$. In [4] (where $A=B=1$, and $a_{0}=b_{0}$ ) the formula

$$
\left\langle\begin{array}{l}
n \\
k
\end{array}\right\rangle=\sum_{i=0}^{n-k}\left(\begin{array}{c}
n-i \\
k
\end{array}\right) \delta_{i}^{a}+\sum_{j=0}^{k}\left(\begin{array}{l}
n-j \\
k-j
\end{array}\right) \delta_{j}^{b}-\left(\begin{array}{l}
n \\
k
\end{array}\right) a_{0}
$$

was established, where $\delta_{i}^{a}=a_{i}-a_{i-1}$ for positive $i$, and $\delta_{0}^{a}=a_{0}$ (analogous scheme holds for the sequence $\left\{b_{n}\right\}$ ). At the first sight (1.3) is strange because it contains $a_{0}$, meanwhile the structure says no influence of $a_{0}=b_{0}$ on the triangle. But there is no contradiction, since a short calculation shows that we can exclude $a_{0}$ (and $\delta_{0}^{a}, \delta_{0}^{b}$ ) from (1.3). Moreover it is easy to see that (1.2) and (1.3) are compatible. Indeed, by (1.3) we have

$$
\left\langle\begin{array}{l}
n \\
k
\end{array}\right\rangle=\sum_{i=1}^{n-k}\left(\begin{array}{c}
n-i \\
k
\end{array}\right)\left(a_{i}-a_{i-1}\right)+\left(\begin{array}{l}
n \\
k
\end{array}\right) b_{0}+\sum_{j=1}^{k}\left(\begin{array}{l}
n-j \\
k-j
\end{array}\right)\left(b_{j}-b_{j-1}\right),
$$

and for $1 \leq k \leq n-1$, via $a_{0}=b_{0}$ it leads to

$$
\left\langle\begin{array}{l}
n \\
k
\end{array}\right\rangle=\sum_{i=1}^{n-k}\left(\begin{array}{c}
n-1-i \\
k-1
\end{array}\right) a_{i}+\sum_{j=1}^{k}\left(\begin{array}{c}
n-1-j \\
k-j
\end{array}\right) b_{j}
$$

The next frame collects some relevant Pascal type triangles (or arrays) have been already studied.

\begin{tabular}{|c|c|l|}
\hline$\left\{a_{n}\right\}$ & $\left\{b_{n}\right\}$ & \multicolumn{1}{|c|}{ Reference } \\
\hline 1 & 1 & Pascal Triangle (PT) \\
\hline$A^{n}$ & $B^{n}$ & Raab [11] (AB-based PT) \\
\hline 2 & 1 & Hosoya [8] (Asymmetrical PT) \\
\hline arbitrary & arbitrary & Ensley [4] (GAT) \\
\hline$F_{n+1}$ & $F_{n+1}$ & Ensley [4] (shifted Fibonacci Triangle) \\
\hline 0 & $\frac{1}{n}$ & Dil - Mező [3] (Hyperharmonic numbers) \\
\hline 0 & $F_{n}$ & Dil - Mezó [3] (Hyper-Fibonacci numbers) \\
\hline$F_{2 n-1}$ & $F_{n-1}$ & Dil - Mezó [3] \\
\hline$A^{n} a$ & $B^{n} b$ & Belbachir - Szalay [2] \\
\hline
\end{tabular}

Assume now that $A=B=1$. Then the rectangular shape matrix $\mathbf{M}$ and the triangular shape GAT differ only in their appearance. Indeed, apart from the geometrical display, the identity

$$
M_{k, n}=\left\langle\begin{array}{c}
k+n \\
n
\end{array}\right\rangle
$$

transmits them to each other for $k+n \geq 1$. Apparently, for $k \geq 1$ and $n \geq 1$ the formulas (1.2) and (1.1) are equivalent via (1.4). Really, replacing $n$ by $k+n$ and $k$ by $n$ in (1.2) at the same time we arrive at (1.1). 
Obviously, with the parameters $A$ and $B$ a kind of Generalized Pascal Array (GPA, given by the matrix $\widetilde{\mathbf{M}}$ ) can be obtained if we introduce the modification

$$
\widetilde{M}_{k, 0}=A^{k} a_{k}, \quad \widetilde{M}_{0, n}=B^{n} b_{n}, \quad k \geq 1, n \geq 1,
$$

and

$$
\widetilde{M}_{k, n}=B \widetilde{M}_{k, n-1}+A \widetilde{M}_{k-1, n}, \quad k n \neq 0 .
$$

In the sequel we always assume that $A=B=1$, and in this paper, we basicly investigate the situation, when the sequences $\left\{a_{n}\right\}=\left\{b_{n}\right\}$ are the Fibonacci, or the Lucas sequence. When $a_{n}=b_{n}=F_{n+1}$, as a consequence of (1.3), Ensley provided

$$
\sum_{i=1}^{n-k}\left(\begin{array}{c}
n-i \\
k
\end{array}\right) F_{i-2}+\sum_{j=1}^{k}\left(\begin{array}{l}
n-j \\
k-j
\end{array}\right) F_{j-2}+\left(\begin{array}{l}
n \\
k
\end{array}\right),
$$

for the $k^{t h}$ entry in row $n$, we develope a more informative explicit formula. In this study, we also show a new and simple proof for one of the equivalent formulas (1.2), (1.1) and (1.3). Note that in [4] the author used a combination of two preliminary lemmata, in [3] and in [2] the technique of induction was used. Here we apply an elementary "atomic" observation. Later we will use some preliminary lemmata, which are stated here.

1.1. Lemma. For arbitrary nonnegative integer $n$ we have

$$
\begin{aligned}
\sum_{i=0}^{n}\left(\begin{array}{c}
n \\
i
\end{array}\right) F_{i} & =F_{2 n}, \\
\sum_{i=0}^{n}(-1)^{i}\left(\begin{array}{c}
n \\
i
\end{array}\right) F_{i} & =-F_{n} .
\end{aligned}
$$

Proof. See [9], Theorems 12.5 and 12.6, on pages $157-158$.

We note that in the case of the second statement of Lemma 1.1, some inaccuracy appears in [9].

1.2. Lemma. Let $n$ be a nonnegative integer. Then

$$
\begin{aligned}
\sum_{i=0}^{n}\left(\begin{array}{c}
n \\
i
\end{array}\right) L_{i} & =L_{2 n}, \\
\sum_{i=0}^{n}(-1)^{i}\left(\begin{array}{c}
n \\
i
\end{array}\right) L_{i} & =L_{n}
\end{aligned}
$$

hold.

Proof. See [9], remarks after Theorems 12.5 and 12.6, on pages 157-158.

1.3. Lemma. Let $n, n_{1}$ and $n_{2}$ be nonnegative integers. Then

$$
\sum_{i=0}^{n}\left(\begin{array}{c}
n-i \\
n_{1}
\end{array}\right)\left(\begin{array}{c}
i \\
n_{2}
\end{array}\right)=\left(\begin{array}{c}
n+1 \\
n_{1}+n_{2}+1
\end{array}\right)
$$

is valid.

Proof. This is a corollary of the Vandermonde identity. 


\section{The "atomic" lemma, the principle of superposition, and some easily computable arithmetic triangles}

Recall, that $A=B=1$. Let $\left\langle\begin{array}{l}n \\ k\end{array}\right\rangle_{\left\{a_{n}, b_{n}\right\}}$ denote the elements of the Generalized Pascal Triangle (in short GPT) generated by the sequences $\left\{a_{n}\right\}$ and $\left\{b_{n}\right\}$. For every given sequences $\left\{a_{n}\right\},\left\{b_{n}\right\},\left\{c_{n}\right\}$ and $\left\{d_{n}\right\}$, we can show easily that

$$
\left\langle\begin{array}{l}
n \\
k
\end{array}\right\rangle_{\left\{a_{n}, b_{n}\right\}}+\left\langle\begin{array}{l}
n \\
k
\end{array}\right\rangle_{\left\{c_{n}, d_{n}\right\}}=\left\langle\begin{array}{l}
n \\
k
\end{array}\right\rangle_{\left\{a_{n}+c_{n}, b_{n}+d_{n}\right\}} .
$$

Now we intend to split this effect into elementary parts, and as a consequence we describe a method for determining $\left\langle\begin{array}{l}n \\ k\end{array}\right\rangle$ when the modifying sequences $\left\{c_{n}\right\}$ and $\left\{d_{n}\right\}$ are simple from one sort of point of view. The next lemma describes the elementary situation when an existing GPT is modified by $c \in \mathbb{R}$ at exactly one element of one of the legs. That is, apart from one entry of $\left\{c_{n}\right\}$ or $\left\{d_{n}\right\}$ we assume $c_{n}=d_{n}=0$. Clearly, such a modification can be applied as many times as we need, and the influences of the consecutive modifications can be superposed. At the end of the section we will see, that this approach is not sufficient to handle the case of Fibonacci, and the Lucas triangle.

Assume that there is given a GPT by the sequences $\left\{a_{n}\right\}$ and $\left\{b_{n}\right\}$.

2.1. Lemma. If one modifies the element $\left\langle{ }_{j}^{i}\right\rangle_{\left\{a_{n}, b_{n}\right\}}$ located on one of the legs of a triangle $(i \geq 1$, further $j=0$ or $j=i$ ) by adding $c \in \mathbb{R}$ to, then the only change on the legs is $\left\langle\begin{array}{l}i \\ j\end{array}\right\rangle_{\text {new }}=\left\langle\begin{array}{l}i \\ j\end{array}\right\rangle_{\left\{a_{n}, b_{n}\right\}}+c$. Further, in the inner part of the triangle we find

(1) in case of $j=0$ (left leg)

$$
\left\langle\begin{array}{l}
n \\
k
\end{array}\right\rangle_{n e w}= \begin{cases}\left\langle\begin{array}{l}
n \\
k
\end{array}\right\rangle_{\left\{a_{n}, b_{n}\right\}}+c\left(\begin{array}{c}
n-i-1 \\
k-1
\end{array}\right), & \text { if } n \geq i+1 \text { and } 1 \leq k \leq n-i \\
\left\langle\begin{array}{l}
n \\
k
\end{array}\right\rangle_{\left\{a_{n}, b_{n}\right\}}, & \text { otherwise, }\end{cases}
$$

(2) in case of $j=i$ (right leg)

$$
\left\langle\begin{array}{l}
n \\
k
\end{array}\right\rangle_{n e w}= \begin{cases}\left\langle\begin{array}{l}
n \\
k
\end{array}\right\rangle_{\left\{a_{n}, b_{n}\right\}}+c\left(\begin{array}{c}
n-i-1 \\
k-i
\end{array}\right), & \text { if } n \geq i+1 \text { and } i \leq k \leq n-1 \\
\left\langle\begin{array}{l}
n \\
k
\end{array}\right\rangle_{\left\{a_{n}, b_{n}\right\}}, & \text { otherwise. }\end{cases}
$$

Proof. It is obvious by the construction (see Figure 1, as an illustration with $i=2$, $j=0)$.

We can build simple GPT's by starting with the empty triangle (any $\left\langle\begin{array}{l}n \\ k\end{array}\right\rangle$ is zero), using element by element of the sequences $\left\{c_{n}\right\}$ and $\left\{d_{n}\right\}$. Note, that the idea is more and less due to Ensley [4], although he started with the classical Pascal triangle, therefore at the end of the procedure he removed that. Observe, that only inserting $c_{1}, c_{2}, \ldots, c_{n-k}$, and $d_{1}, d_{2}, \ldots, d_{k}$ has influence on the element $\left\langle\begin{array}{l}n \\ k\end{array}\right\rangle$. Thus, by Lemma 2.1 and the principle of superposition we obtain immediately a (new) proof for the identity $(1.2)$, since $\left(\begin{array}{l}n \\ k\end{array}\right)_{\Gamma}=\left(\begin{array}{l}n \\ k\end{array}\right)$ holds if $k$ is nonnegative.

Now we demonstrate the applicabilty of Lemma 2.1 by a few further examples. Locally we use the notations

$$
\Lambda_{c}=\sum_{i=1}^{n-k}\left(\begin{array}{c}
n-1-i \\
k-1
\end{array}\right) c_{i} \quad \text { and } \quad \Lambda_{d}=\sum_{i=1}^{k}\left(\begin{array}{c}
n-1-i \\
n-k-1
\end{array}\right) d_{i}
$$

Clearly, we have

$$
\left\langle\begin{array}{l}
n \\
k
\end{array}\right\rangle_{n e w}=\left\langle\begin{array}{l}
n \\
k
\end{array}\right\rangle_{\text {old }}+\Lambda_{c}+\Lambda_{d}
$$




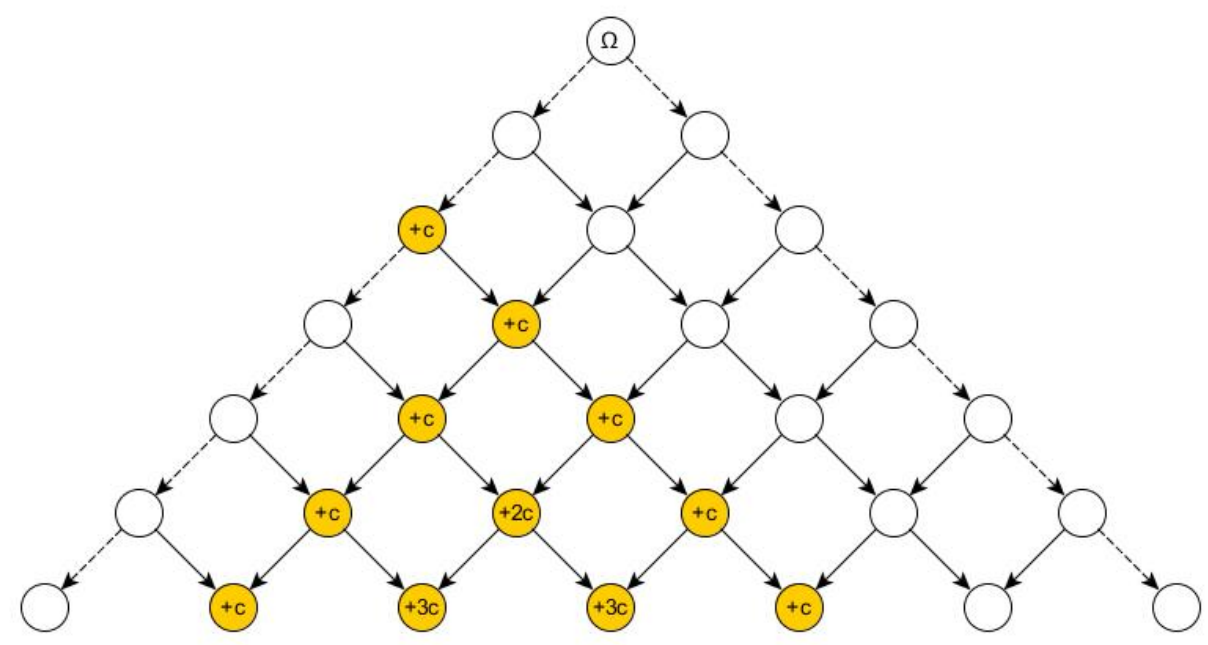

Figure 1. The influence of modification of one leg element

We will also use Lemma 1.3 to calculate the appropriate sums.

(1) Initial triangle: empty; modified by $c_{n}=d_{n}=1$ (case of classical Pascal triangle).

$\left\langle\begin{array}{l}n \\ k\end{array}\right\rangle_{\text {new }}=\sum_{i=1}^{n-k}\left(\begin{array}{c}n-1-i \\ k-1\end{array}\right)+\sum_{i=1}^{k}\left(\begin{array}{l}n-1-i \\ n-k-1\end{array}\right)=\left(\begin{array}{c}n-1 \\ k\end{array}\right)+\left(\begin{array}{l}n-1 \\ n-k\end{array}\right)=\left(\begin{array}{l}n \\ k\end{array}\right)$.

(2) Initial triangle: empty; modified by $c_{n}=d_{n}=p(n)$, where $p(x)$ is a given polynomial of degree $d \geq 1$. First we express the polynomial as a linear combination of the binomial coefficients $\left(\begin{array}{c}x \\ i\end{array}\right), i=1, \ldots d$. Then we apply Lemma 1.3 to determine the sums appearing as the influence of the left, and the right leg. For instance, put $p(x)=x^{2}$, so $c_{n}=d_{n}=n^{2}$. Since $x^{2}=2\left(\begin{array}{l}x \\ 2\end{array}\right)+\left(\begin{array}{l}x \\ 1\end{array}\right)$, we find

$\Lambda_{c}=\sum_{i=1}^{n-k}\left(\begin{array}{c}n-1-i \\ k-1\end{array}\right) i^{2}=\sum_{i=1}^{n-k}\left(\begin{array}{c}n-1-i \\ k-1\end{array}\right)\left(2\left(\begin{array}{l}i \\ 2\end{array}\right)+\left(\begin{array}{l}i \\ 1\end{array}\right)\right)$

$=2 \sum_{i=1}^{n-k}\left(\begin{array}{c}n-1-i \\ k-1\end{array}\right)\left(\begin{array}{l}i \\ 2\end{array}\right)+\sum_{i=1}^{n-k}\left(\begin{array}{c}n-1-i \\ k-1\end{array}\right)\left(\begin{array}{l}i \\ 1\end{array}\right)=2\left(\begin{array}{c}n \\ k+2\end{array}\right)+\left(\begin{array}{c}n \\ k+1\end{array}\right)$.

Similarly,

$$
\Lambda_{d}=2\left(\begin{array}{c}
n \\
k-2
\end{array}\right)+\left(\begin{array}{c}
n \\
k-1
\end{array}\right)
$$

thus

$$
\left\langle\begin{array}{l}
n \\
k
\end{array}\right\rangle_{n e w}=2\left(\begin{array}{c}
n \\
k-2
\end{array}\right)+\left(\begin{array}{c}
n \\
k-1
\end{array}\right)+\left(\begin{array}{c}
n \\
k+1
\end{array}\right)+2\left(\begin{array}{c}
n \\
k+2
\end{array}\right) .
$$

(3) Initial triangle: Pascal triangle; modified by $c_{n}=1(n \in \mathbb{N}$, Asymmetrical PT in [8], see Figure 2). Only the changes at $\left(\begin{array}{l}1 \\ 0\end{array}\right),\left(\begin{array}{l}2 \\ 0\end{array}\right), \ldots,\left(\begin{array}{c}n-k \\ 0\end{array}\right)$ cause variation at 
$\left(\begin{array}{l}n \\ k\end{array}\right)$. By Lemma 2.1 we find

$$
\left\langle\begin{array}{l}
n \\
k
\end{array}\right\rangle_{n e w}= \begin{cases}\left(\begin{array}{l}
n \\
k
\end{array}\right)+\sum_{i=1}^{n-k}\left(\begin{array}{c}
n-i-1 \\
k-1
\end{array}\right)=\left(\begin{array}{c}
n \\
k
\end{array}\right)+\left(\begin{array}{c}
n-1 \\
k
\end{array}\right), & \text { if } n \geq 2 \text { and } 1 \leq k \leq n-1 \\
\left(\begin{array}{l}
n \\
n
\end{array}\right)=1, & \text { if } k=n,\end{cases}
$$

which coincides the result of [8].

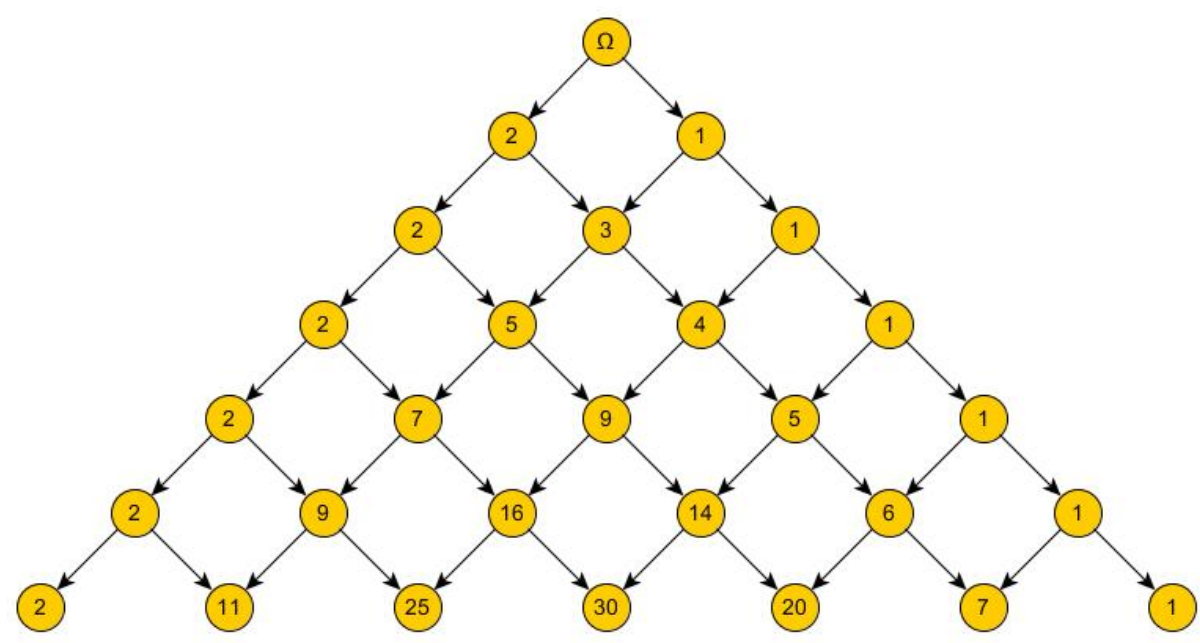

Figure 2. Asymmetric Pascal triangle

Suppose now that we want to modify the empty triangle by the sequences $c_{n}=d_{n}=$ $F_{n}$. Then we face, among others, to the problem of determining the sum

$$
\Lambda_{c}=\sum_{i=1}^{n-k}\left(\begin{array}{c}
n-1-i \\
k-1
\end{array}\right) F_{i}
$$

but unfortunately there is no closed formula to express it. Subsequently, we need something else to describe the Fibonacci Pascal triangle.

\section{Fibonacci and Lucas triangles}

3.1. Fibonacci triangle. In this part, first we focus on the Fibonacci triangle, which was introduced by Ensley [4]. Recall, that he took $c_{n}=d_{n}=F_{n+1}$. Denoting the elements of this triangle by $\left\langle\begin{array}{l}n \\ k\end{array}\right\rangle_{F_{n+1}}$, Ensley showed

$$
\left\langle\begin{array}{l}
n \\
k
\end{array}\right\rangle_{F_{n+1}}=\left(\begin{array}{l}
n \\
k
\end{array}\right)+\sum_{i=1}^{n-k}\left(\begin{array}{c}
n-i \\
k
\end{array}\right) F_{i-2}+\sum_{j=1}^{n-k}\left(\begin{array}{l}
n-j \\
k-j
\end{array}\right) F_{j-2}
$$

At the end of this section we will give a more applicable formula for the elements of this triangle, but now we start with studying the triangle generated by $a_{n}=b_{n}=F_{n}$ (see Figure 3). The main result is the following.

3.1. Theorem. For any nonnegative integers $n$ and $k$ we have

$$
\left\langle\begin{array}{l}
n \\
k
\end{array}\right\rangle_{F_{n}}=F_{n+k}-q_{k}(n)
$$


where

$$
q_{k}(x)=2 \sum_{j=0}^{\lfloor k / 2\rfloor}\left(\begin{array}{c}
x \\
k-2 j
\end{array}\right) F_{2 j}
$$

is a rational polynomial of degree $k-2$ if $k \geq 2$, and $q_{0}(x)=q_{1}(x)=0$.

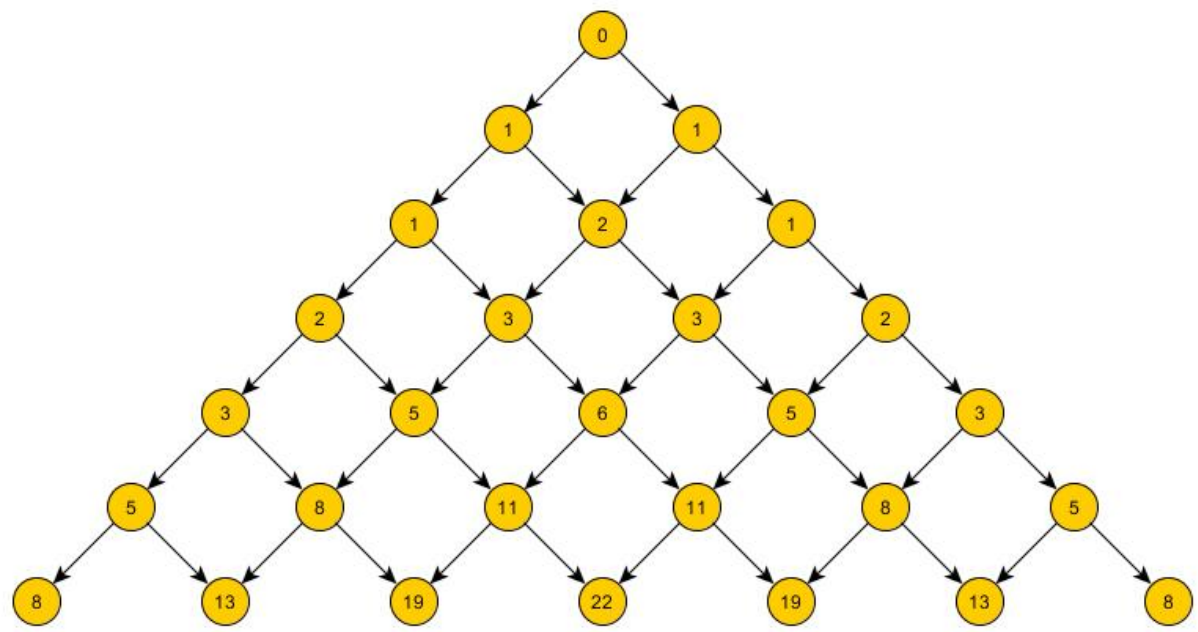

Figure 3. Fibonacci Pascal triangle

For proving Theorem 3.1, we need

3.2. Lemma. For any nonnegative integer $k$, the specific value $q_{k}(k)$ is given by $q_{k}(k)=$ $F_{2 k}-F_{k}$.

Proof.

$$
q_{k}(k)=2 \sum_{j=0}^{\lfloor k / 2\rfloor}\left(\begin{array}{c}
k \\
k-2 j
\end{array}\right) F_{2 j}=2 \sum_{j=0}^{\lfloor k / 2\rfloor}\left(\begin{array}{c}
k \\
2 j
\end{array}\right) F_{2 j}=\sum_{j=0}^{k}\left(\begin{array}{l}
k \\
j
\end{array}\right) F_{j}+\sum_{j=0}^{k}(-1)^{j}\left(\begin{array}{l}
k \\
j
\end{array}\right) F_{j} .
$$

It implies, by Lemma 1.1, that $q_{k}(k)=F_{2 k}-F_{k}$.

3.3. Lemma. The equality $q_{k}(N+1)-q_{k}(N)=q_{k-1}(N)$ fulfils for any nonnegative integers $N$ and $k$.

Proof. Suppose first that $k=2 \kappa$. Then

$$
\begin{aligned}
\frac{q_{k}(N+1)}{2} & =\sum_{j=0}^{\kappa}\left(\begin{array}{c}
N+1 \\
k-2 j
\end{array}\right) F_{2 j}=\sum_{j=0}^{\kappa-1}\left(\left(\begin{array}{c}
N \\
k-2 j-1
\end{array}\right)+\left(\begin{array}{c}
N \\
k-2 j
\end{array}\right)\right) F_{2 j}+\left(\begin{array}{c}
N \\
0
\end{array}\right) F_{k} \\
& =\sum_{j=0}^{\kappa-1}\left(\begin{array}{c}
N \\
k-2 j-1
\end{array}\right) F_{2 j}+\sum_{j=0}^{\kappa}\left(\begin{array}{c}
N \\
k-2 j
\end{array}\right) F_{2 j}=\frac{q_{k-1}(N)}{2}+\frac{q_{k}(N)}{2} .
\end{aligned}
$$


If $k=2 \kappa+1$ is odd, then similarly we get

$$
\begin{aligned}
\frac{q_{k}(N+1)}{2} & =\sum_{j=0}^{\kappa}\left(\begin{array}{c}
N+1 \\
k-2 j
\end{array}\right) F_{2 j}=\sum_{j=0}^{\kappa}\left(\left(\begin{array}{c}
N \\
k-2 j-1
\end{array}\right)+\left(\begin{array}{c}
N \\
k-2 j
\end{array}\right)\right) F_{2 j} \\
& =\sum_{j=0}^{\kappa}\left(\begin{array}{c}
N \\
k-2 j-1
\end{array}\right) F_{2 j}+\sum_{j=0}^{\kappa}\left(\begin{array}{c}
N \\
k-2 j
\end{array}\right) F_{2 j}=\frac{q_{k-1}(N)}{2}+\frac{q_{k}(N)}{2} .
\end{aligned}
$$

Now we turn to the proof of Theorem 3.1.

Proof. First we show the statement for the legs of the GPT.

$$
\begin{aligned}
& \left\langle\begin{array}{l}
n \\
0
\end{array}\right\rangle_{F_{n}}=F_{n}-q_{0}(n)=F_{n}, \\
& \left\langle\begin{array}{l}
n \\
n
\end{array}\right\rangle_{F_{n}}=F_{2 n}-q_{n}(n)=F_{2 n}-\left(F_{2 n}-F_{n}\right)=F_{n} .
\end{aligned}
$$

Now assume that $n \geq 2$ and $1 \leq k \leq n-1$. After verifying $\left\langle\begin{array}{l}2 \\ 1\end{array}\right\rangle_{F_{n}}=F_{3}-q_{1}(2)=2$, we use the technique of induction. Hence we assume that (3.1) is true for $n \leq N(N \geq 2)$. Applying it, together with Lemma 3.3, we deduce

$$
\begin{aligned}
\left\langle\begin{array}{c}
N+1 \\
k
\end{array}\right\rangle_{F_{n}} & =\left\langle\begin{array}{c}
N \\
k-1
\end{array}\right\rangle_{F_{n}}+\left\langle\begin{array}{l}
N \\
k
\end{array}\right\rangle_{F_{n}}=\left(F_{N+k-1}-q_{k-1}(N)\right)+\left(F_{N+k}-q_{k}(N)\right) \\
& =F_{N+k-1}+F_{N+k}-\left(q_{k-1}(N)-q_{k}(N)\right)=F_{N+k+1}-q_{k}(N+1) .
\end{aligned}
$$

Since the polynomials $q_{k}(x)$ play crucial role in (3.1), in the next table we give the first few of them explicitly. Recall, that $q_{0}(x)=q_{1}(x)=0$.

\begin{tabular}{|c|c|c|c|c|c|}
\hline$k$ & 2 & 3 & 4 & 5 & 6 \\
\hline$q_{k}(x)$ & 2 & $2 x$ & $x^{2}-x+6$ & $\frac{1}{3}\left(x^{3}-3 x^{2}+20 x\right)$ & $\frac{1}{12}\left(x^{4}-6 x^{3}+47 x^{2}-42 x+192\right)$ \\
\hline
\end{tabular}

The proof of Lemma 3.3 gives a hint how to determine $q_{k-1}(x)$ if one knows $q_{k}(x)$. The reverse order is more interesting since we know the beginning of the list $q_{2}(x), q_{3}(x)$, ... etc. Although we have (3.2) in Theorem 3.1, it may be challenging to know how to generate the next unknown element of the list. Suppose that the polynomial

$$
q_{t+1}(x)=b_{t-1} x^{t-1}+b_{t-2} x^{t-2}+\cdots+b_{1} x+b_{0}
$$

is known, and we intend to determine the coefficients $a_{i}$ of the polynomial

$$
q_{t+2}(x)=a_{t} x^{t}+a_{t-1} x^{t-1}+\cdots+a_{1} x+a_{0} .
$$


By Lemma 3.3, we expand the difference $q_{t+2}(x+1)-q_{t+2}(x)$, and compare it to $q_{t+1}(x)$. Hence we must consider the system of equations

$$
\begin{aligned}
b_{t-1} & =\left(\begin{array}{l}
t \\
1
\end{array}\right) a_{t}, \\
b_{t-2} & =\left(\begin{array}{l}
t \\
2
\end{array}\right) a_{t}+\left(\begin{array}{c}
t-1 \\
1
\end{array}\right) a_{t-1}, \\
b_{t-3} & =\left(\begin{array}{l}
t \\
3
\end{array}\right) a_{t}+\left(\begin{array}{c}
t-1 \\
2
\end{array}\right) a_{t-1}+\left(\begin{array}{c}
t-2 \\
1
\end{array}\right) a_{t-2}, \\
& \vdots \\
b_{1} & =\left(\begin{array}{c}
t \\
t-1
\end{array}\right) a_{t}+\left(\begin{array}{l}
t-1 \\
t-2
\end{array}\right) a_{t-1}+\cdots+\left(\begin{array}{l}
2 \\
1
\end{array}\right) a_{2}, \\
b_{0} & =\left(\begin{array}{l}
t \\
t
\end{array}\right) a_{t}+\left(\begin{array}{c}
t-1 \\
t-1
\end{array}\right) a_{t-1}+\cdots+\left(\begin{array}{l}
2 \\
2
\end{array}\right) a_{2}+\left(\begin{array}{l}
2 \\
2
\end{array}\right) a_{2} .
\end{aligned}
$$

From the top of the list of equations to down one can consecutively determine the coefficients $a_{t}, a_{t-1}, \ldots, a_{1}$. Then the constant term $a_{0}$ follows from the equality $q_{t+2}(t+2)=F_{2 t+4}-F_{t+2}$.

At the end of this section recall that $\sum_{k=0}^{n}\left\langle\begin{array}{l}n \\ k\end{array}\right\rangle_{F_{n}}=2^{n+1}-2 F_{n+1}$ ([2], after Example 1). Combining the former expression with (3.1), we have the following

3.4. Corollary. For any nonnegative integer $n$, the identity

$$
\sum_{k=0}^{n} q_{k}(n)=F_{2 n+2}+F_{n+1}-2^{n+1}
$$

holds.

The nonexistence of closed form for (2.1) was the motivation to work out a different approach for Fibonacci Pascal triangle. For the specific case $n=2 k$ we see, that the two sums in (1.3) coincide. This observation, together with Theorem 3.1 implies

3.5. Corollary. If $k$ is a positive integer, then we get

$$
\sum_{i=1}^{k}\left(\begin{array}{c}
2 k-1-i \\
k-1
\end{array}\right) F_{i}=\frac{F_{3 k}-q_{k}(2 k)}{2} .
$$

3.2. Lucas Pascal triangle. After studying Fibonacci Pascal triangle, it is natural to consider Lucas Pascal triangle, i.e. when $a_{n}=b_{n}=L_{n}$ is the $n^{t h}$ term of the Lucas sequence $\left\{L_{n}\right\}_{n=0}^{\infty}$ (see Figure 4$)$.

Without detailing the proofs (only follow the maintance of Fibonacci PT), we yield the main result and the corresponding lemmata.

3.6. Theorem. For any nonnegative integers $n$ and $k$, we have

$$
\left\langle\begin{array}{l}
n \\
k
\end{array}\right\rangle_{L_{n}}=L_{n+k}-r_{k}(n)
$$

where

$$
r_{k}(x)=2 \sum_{j=0}^{\lfloor(k-1) / 2\rfloor}\left(\begin{array}{c}
x \\
k-1-2 j
\end{array}\right) F_{2 j+1}
$$




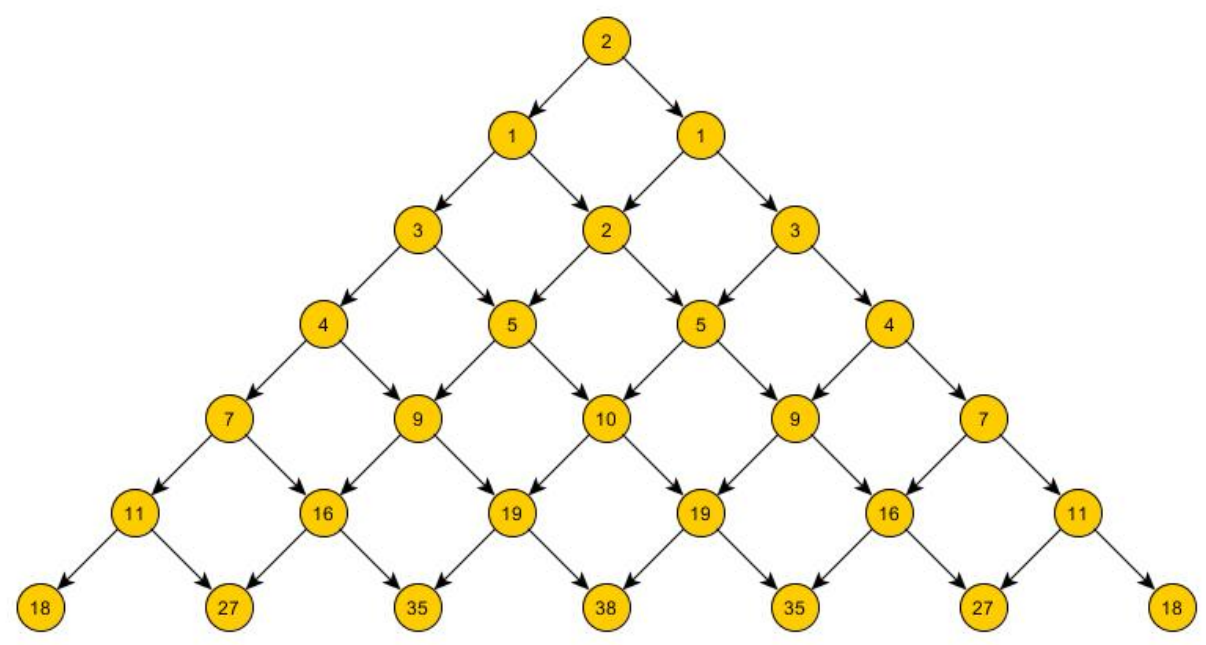

Figure 4. Lucas Pascal triangle

is a rational polinomial of degree $k-1$ if $k \geq 1$, and $r_{0}(x)=0$.

Proof. Without going into details, we follow the method used in the case of $a_{n}=b_{n}=$ $F_{n}$.

The lemmata we need are the following.

3.7. Lemma. The equality $r_{k}(k)=L_{2 k}-L_{k}$ holds for any nonnegative integer $k$.

3.8. Lemma. For any nonnegative integers $N$ and $k$, we have $r_{k}(N+1)-r_{k}(N)=$ $r_{k-1}(N)$.

The first few polynomials $r_{k}(x)$ are listed here.

\begin{tabular}{|c|c|c|c|c|c|}
\hline$k$ & 1 & 2 & 3 & 4 & 5 \\
\hline$r_{k}(x)$ & 2 & $2 x$ & $x^{2}-x+8$ & $\frac{1}{3}\left(x^{3}-3 x^{2}+26 x\right)$ & $\frac{1}{12}\left(x^{4}-6 x^{3}+59 x^{2}-54 x+264\right)$ \\
\hline
\end{tabular}

3.3. Ensley's Fibonacci Triangle and a generalization. Now we are ready to handle Ensley's Fibonacci Triangle, when $a_{n}=b_{n}=F_{n+1}$, by exploiting the results on Fibonacci and Lucas Triangles.

Assume generally, that the sequence $\left\{G_{n}\right\}$ satisfies the recursive rule

$$
G_{n}=G_{n-1}+G_{n-2} \quad(n \geq 2)
$$

with the initial values $G_{0}$ and $G_{1}$. It is well known, that such a sequence can be given by a linear combination of any two linearly independent recurrences (like Fibonacci and Lucas sequences), which satisfy (3.3). Taking the Fibonacci and Lucas sequences as basis, the solution of the vector equation

$$
\left[\begin{array}{l}
F_{0} \\
F_{1}
\end{array}\right] x+\left[\begin{array}{l}
L_{0} \\
L_{1}
\end{array}\right] y=\left[\begin{array}{l}
G_{0} \\
G_{1}
\end{array}\right]
$$

(via $F_{0}=0, F_{1}=1, L_{0}=2$ and $L_{1}=1$ ) is $y=G_{0} / 2, x=\left(2 G_{1}-G_{0}\right) / 2$. That is,

$$
G_{n}=\frac{2 G_{1}-G_{0}}{2} F_{n}+\frac{G_{0}}{2} L_{n}
$$


and, by Theorems 3.1 and 3.6, and the principle of superposition we have the following general theorem.

3.9. Theorem. The GPT generated by $a_{n}=b_{n}=G_{n}$ satisfies

$$
\left\langle\begin{array}{l}
n \\
k
\end{array}\right\rangle_{G_{n}}=G_{n+k}-\frac{\left(2 G_{1}-G_{0}\right) q_{k}(n)+G_{0} r_{k}(n)}{2} .
$$

Specifying $G_{n}=F_{n+1}$, we have

$$
\left[\begin{array}{l}
G_{0} \\
G_{1}
\end{array}\right]=\left[\begin{array}{l}
F_{1} \\
F_{2}
\end{array}\right]=\left[\begin{array}{l}
1 \\
1
\end{array}\right]
$$

hence now $x=y=1 / 2$. Thus $F_{n+1}=\left(F_{n}+L_{n}\right) / 2$, and we conclude

3.10. Corollary. For any nonnegative integers $n$ and $k$, we conclude

$$
\left\langle\begin{array}{l}
n \\
k
\end{array}\right\rangle_{F_{n+1}}=F_{n+k+1}-\frac{q_{k}(n+1)+r_{k}(n+1)}{2} .
$$

Acknowledgements. We thank the referee for valuable comments that greatly improved the quality of this paper.

\section{References}

[1] Abramowitz, M. and Stegun, I. A., Handbook of mathematical functions with formulas, graphs and mathematical tables, Applied Mathematics Series 55 (10 ed.), New York, USA, 1972.

[2] Belbachir, H. and Szalay, L., On the arithmetic triangles, Šiauliai Math. Sem., 9 (17), 15-26, 2014.

[3] Dil, A. and Mező, I., A symmetric algorithm for hyperharmonic and Fibonacci numbers, Appl. Math. Comp., 206, 942-951, 2008.

[4] Ensley, D., Fibonacci's triangle and other abominations, in: The Edge of the Universe: Celebrating Ten Years of Maths Horizons, MAA, 287-307, 2006.

[5] Ferri, G., Faccio, M. and D'Amico, A., A new numerical triangle showing links with Fibonacci numbers, Fibonacci Quart., 29, 316-321, 1991.

[6] Ferri, G., Faccio, M. and D'Amico, A., Fibonacci numbers and ladder network impedance, Fibonacci Quart., 30, 62-67, 1992.

[7] Hosoya, H., Fibonacci triangle, Fibonacci Quart., 14, 173-178, 1976.

[8] Hosoya, H., Pascal's triangle, non-adjacent numbers, and D-dimensional atomic orbitals, J. Math. Chemistry, 23, 169-178, 1998.

[9] Koshy, T., Fibonacci and Lucas Numbers with Applications, Pure and Applied Mathematics, A Wiley-Interscience Series of Texts, Monographs, and Tracts, 2001.

[10] Pascal, B., Traité du Triangle Arithmétique, avec quelques autres petitez sur la mesme matiere, Paris, 1665.

[11] Raab, J. A., A generalization of the connection between the Fibonacci sequence and Pascal's triangle, Fibonacci Quart., 1, 21-31, 1963.

[12] Sun, Y., Numerical triangles and several classical sequences, Fibonacci Quart., 43, 359-370, 2005. 